\title{
Long term variability in solar wind velocity and IMF intensity and the relationship between solar wind parameters \& geomagnetic activity
}

\author{
G. K. Rangarajan and L. M. Barreto
}

Observatorio Nacional São Cristovao, 29021-400 Rio de Janeiro, Brazil

(Received May 6, 1999; Revised November 8, 1999; Accepted November 8, 1999)

\begin{abstract}
A study is carried out on the mean monthly values of in situ observations of solar wind velocity $(V)$ and the intensity of interplanetary magnetic field, $B$ to elucidate their long term variations using the technique of singular spectrum analysis. It is shown that $B$ exhibits a clear solar cycle signal with progressively deepening minimum and a well-defined longer period variation but $V$ is marked by a $\sim 9$-yr cycle. Time variations in the amplitude of 16-month periodicity, observed sporadically in the velocity earlier by others are clearly brought out, as also the characteristic amplitude changes in a 42-month signal in velocity. It appears that time intervals which show certain significant fluctuations in $B$ are marked by the absence of similar signal in $V$.

$a a$ index is next used as a proxy for solar wind velocity, after establishing a good correlation between Ap and observed $V$ so that we could examine the evolution of different periodicities over 14 solar cycles. A significant trend with least value in 1900 and a near-linear rise up to 1960 is shown to be the main feature of the velocity change. The solar cycle component in $V$ lags the solar activity peak by $\sim 22$ months. Streams emanating from coronal holes in the declining phase seem to be the most dominant contributor to the 11-year variation in velocity. The anomalous pattern of changes in $\mathrm{V}$ observed in cycle 20 is not present in any of the other 13 cycles.

Statistical relationships between $V, B$ and plasma density $(N)$ with $A p$ are studied and it is shown that over three solar cycles $(20,21$ and 22$)$ the patterns are almost the same with a slight change observed in cycle 21 . IMF $B$ and $A p$ are linearly related over a wide range of $A p$ values from close to 0 up to about 60 , whereas density/ $A p$ relation appears insignificant. In case of $V$, an initial rapid rise in $V$ causes moderate changes in $A p$ but for velocity in excess of $700 \mathrm{~km} / \mathrm{sec}$, the enhancement in geomagnetic activity is more rapid. From the statistical relation of several other solar wind/IMF parameters, their variability and combinations with $A p$, one sees linear relation for solar wind electric field, $\mathrm{n} / \mathrm{s}$ component of IMF and variability in the components of $B$. An estimate is made of the base level of the magnetosphere, corresponding to quiet levels of geomagnetic activity.
\end{abstract}

\section{Introduction}

In-situ measurements of IMF and solar wind parameters began in late 1962 and now cover more than three solar cycles. The observations have helped in establishing several useful statistical relationships between indices of geomagnetic activity and the causative solar wind parameters (Svalgaard, 1977; Maezawa, 1978; Smart et al., 1979 and others). The data have also been examined for their long term variability in terms of either the 11-year solar cycle component (Feldman et al., 1978; King, 1979; Bieber et al., 1993 and others) or in terms of other quasi-periodic components such as the 1 to 1.3 -year oscillation in solar wind velocity (Wang and Sheeley, 1988; Bolton, 1990; Gazis et al., 1995; Gazis, 1996). From the early observations of solar wind bulk speed, Snyder et al. (1963) were able to establish its close correlation with geomagnetic activity. Crooker et al. (1977) showed that when long term averages (covering duration of 6 months or more) are considered, the correlation between geomagnetic activity and solar wind velocity is indeed very striking. Later, however, Crooker and Gringauz

Copy right (C) The Society of Geomagnetism and Earth, Planetary and Space Sciences (SGEPSS); The Seismological Society of Japan; The Volcanological Society of Japan; The Geodetic Society of Japan; The Japanese Society for Planetary Sciences.
(1993) found that in solar cycle $21, B s V^{2}$ correlated much better with $A p$ rather than $V$ or $V^{2} . B s$ is taken to be $|B z|$ when $B z$ is negative and $=0$ when positive. Geomagnetic activity indices such as $K p, A p$ or $a a$ have been successfully used as proxy for inferring the solar wind speed in the intervals of time not covered by spacecraft observations (Maer and Dessler, 1964; Feynman and Crooker, 1978; Silverman, 1986 and others).

Report of Feynman (1983) contains many details of the US efforts during 1979-1982 in the study of variations of solar wind on time scales of decades to hundreds of years. King (1991), describing the contents of the NSSDC OMNI tape, discussed the long-term solar wind variations and associated data sources. He found that (i) IMF $B$ has a minimum at solar minimum (ii) maximum in $V$ occurs during the declining phase and (iii) density and pressure have minima during solar activity maximum. He also found significant variation in $B$ and $V$ from one cycle to the next. Rickett and Coles (1991) observed that near solar maximum, average wind speeds were uniformly low. Bruno et al. (1994) studied the changes in selected solar wind parameters at $1 \mathrm{AU}$ through two solar activity cycles, separating the data into parts corresponding to fast and slow solar wind.

In the present analysis, we first examine the time varia- 
tions in $V$ and $B$ using the monthly averages of the parameters covering the period 1965 to 1998 July, encompassing three complete solar cycles 1964-76 (cycle No. 20): 19761986 (No. 21) and 1986-1996 (No. 22). The initial two years of spacecraft observations were marked by large gaps and, therefore, are omitted from consideration. In the second part, we establish a dependable linear relation between the index $A p$ of geomagnetic activity and $V$ for the same interval and use this to infer the monthly average solar wind speed from 1932. Further, based on the fact that the correlation between $A p$ and $a a$ values is close to unity (Mayaud, 1980), we use the monthly values of $a a$ index from 1845 as proxy for solar wind velocity. These time series are then subjected to the technique of Singular Spectrum Analysis-SSA (Vautard et al., 1992; Rangarajan and Araki, 1997) to isolate significant spectral components. In the third part, we group days according to the daily values of different IMF/solar wind parameters and suitable combinations thereof in distinct bins of increasing magnitude and compute the corresponding averages of daily $A p$ values to evaluate the stability of the inter-relationships, shown earlier (Svalgaard, 1977; Maezawa, 1978). The significant features of these analyses are discussed.

\section{Data Analysis}

For isolating significant quasi-periodic oscillations in the time series of solar wind velocity and IMF intensity, we use the technique of SSA because it succeeds eminently in isolating noise from signals and provides a clear view of the time variations of the signals through data adaptive filtering (Dettinger et al., 1995). The isolated components are independent of each other and can be expected to describe different causative sources on the Sun. In addition, the relative significance or the percentage variance contributed by each component can also be estimated (see Rangarajan and Iyemori, 1997; Rangarajan, 1998 for details of the methodology and illustrative examples). In SSA, the choice of the parameter $M$, called the viewing window which decides the size of the trajectory matrix, is subjective. Smaller $M$ (relative to the data length) yields more stable estimates while larger $M$ provides greater resolution. In general, fluctuations with periodicity within the length of the window are well resolved (see Vautard et al., 1992). In our analysis, we choose $M=132$ for the in-situ observations of 379 monthly values of $V$ and $B$ which ensures that solar cycle variation and higher frequencies are adequately resolved. The extended data base of $V$ covering the interval 1846 to 1998 is first decimated every 3 months to yield 607 values and $M$ is now chosen to be 44 (again covering 132 months) which ensures highly stable estimates of components. For establishing the statistical relationship between solar wind/IMF parameters or their combinations in different groups and $A p$ index, we use the method of orthogonal polynomials to derive the equations.

The basic data are the mean daily values from the NSSDC OMNI tape and the updates available on the website. There are gaps in the data set and when the missing values were less than 10 days in a month, the monthly means were computed. Otherwise, the data for the month was treated as missing and given a nominal value of $250 \mathrm{~km} / \mathrm{sec}$ for $V$ and $3.5 \mathrm{nT}$ for $B$, only for plotting purposes. Figure 1 shows plots of the monthly values of $V$ and $B$ from Jan. 1965 to July 1998. For

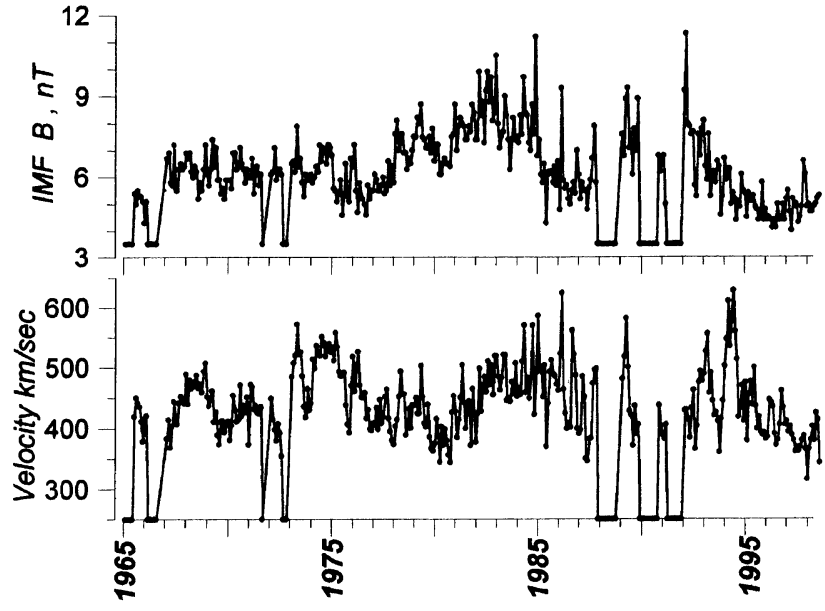

Fig. 1. Mean monthly values of solar wind velocity $(V)$ and the intensity of interplanetary magnetic field $(B)$ between 1965 Jan. and 1998 July. Daily averages from NSSDC OMNI tape are used to derive the monthly values. When data for more than 10 days are missing, corresponding monthly values are shown with a nominal $250 \mathrm{~km} / \mathrm{sec}$ for $V$ and $3.5 \mathrm{nT}$ for $B$.

the purposes of SSA, the missing values were interpolated using the relationship established between $V$ and $A p$. For the study of relationship between $A p$ and the solar wind/IMF parameters, no attempt was made to interpolate any missing daily values.

King (1991) has given complete details of the process of normalization of the data from different spacecrafts and has cautioned that the problem of absolute density calibration has not yet been solved. Density and proton temperature data prior to and after 1971 cannot be compared. Also, large data gaps, when they occur, are not random but are caused by the spacecraft being located inside the magnetosphere. The errors introduced due to this in the monthly mean values are hopefully not significant and will not vitiate the results presented here.

For the velocity in pre-spacecraft era, we use the geomagnetic activity index. We are aware that geomagnetic activity is the result of a complex solar wind-magnetosphere interaction process and that solar wind velocity is only one of the possible contributing factors. We also find that Feynman and Crooker (1978) and Silverman (1986) have used $a a$ index as proxy of solar wind speed. We assume that the long term variability of solar wind velocity discussed here can be considered valid, subject to the uncertainties associated with the proxy nature of the index used to derive the velocity. Though $a a$ index was scaled only from 1868, Nevanlinna and Kataja (1993) were able to successfully extrapolate the index backward in time to 1845 , using the Sodankyla Declination observations and the series of monthly values are now available from July 1844 onwards. Another aspect that may be worthy of emphasis is the fact that $a a$ index is, perhaps, one of the most homogeneous and consistent series (Mayaud, 1980) and, therefore, any systematic bias in the conversion of the magnitude of the index as a measure of velocity will be uniform throughout. 
(a)
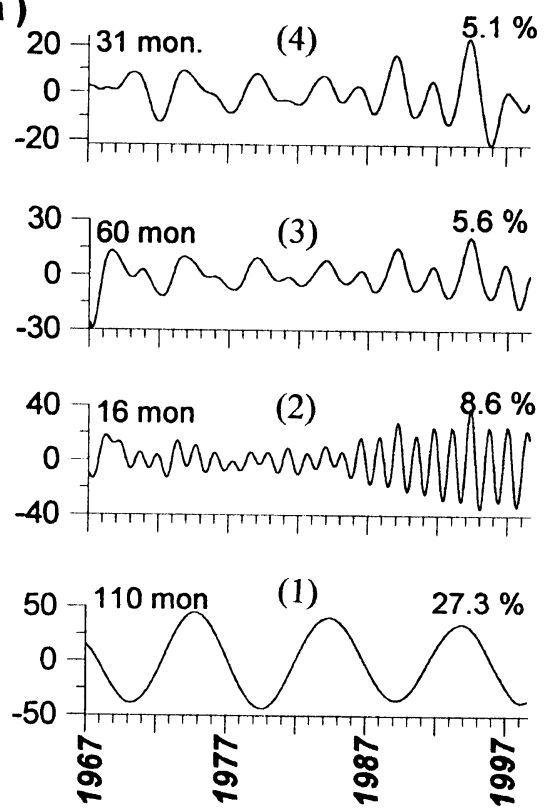

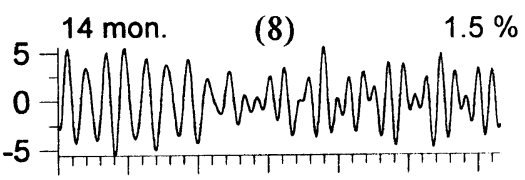

(7)
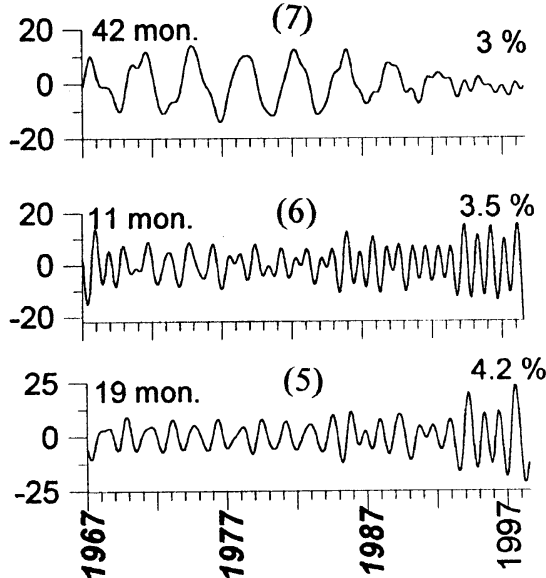

Fig. 2(a). Reconstructed components of $V$ isolated through adaptive filtering by singular spectrum analysis. The periodicity indicated for each is derived from MEM spectrum. The percentage shown in each curve represents the relative contribution of the component to the total variance of the time series.
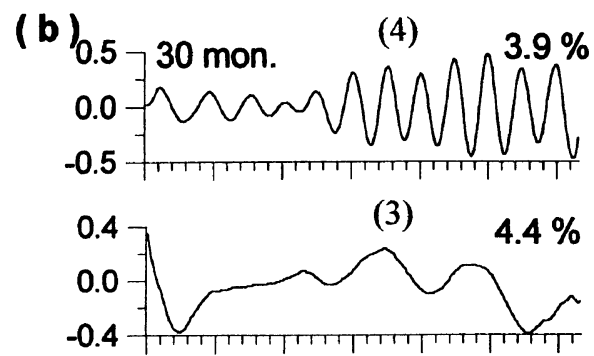

(2)

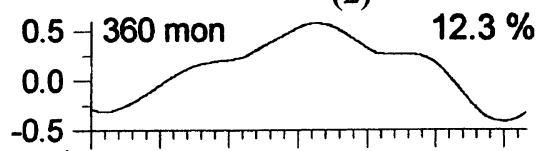

(1)

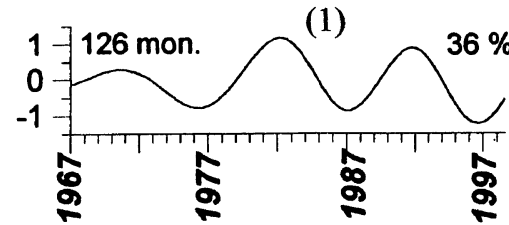

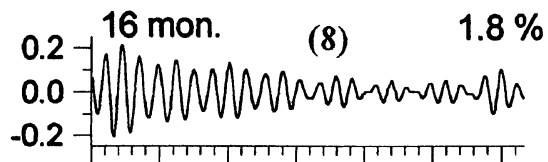
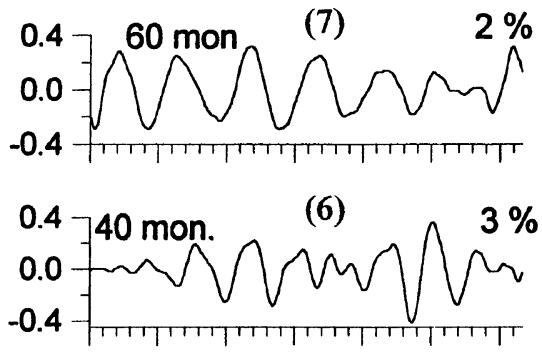

(5)

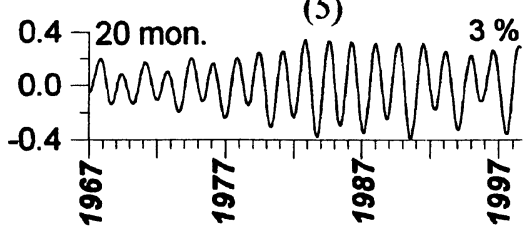

Fig. 2(b). Same as Fig. 2(a), but the results are for IMF $B$.

\section{Results and Discussion}

\subsection{Spacecraft observations (1965-1998)}

Figure 2(a) depicts the time-local properties of the significant reconstructed components (see Rangarajan and Araki, 1997 for derivation of the components) in solar wind velocity, isolated through SSA and in Fig. 2(b) are shown the components isolated from the IMF $B$ time series. The percentage variance accounted for by each is also indicated. It is noticed that about 40 percent of the variability in $V$ or $B$ cannot be accounted for by any persistent features. Periodicity associated with each component was determined using appropriate low order auto-regressive model coefficients using Maximum Entropy Method. Penland et al. (1991) have shown how SSA helps in removing noise from data so that a very low order MEM can provide very high resolution and resolve even very closely-spaced frequency components.

One does not see a solar cycle ( $\sim 11$-year $)$ component in $V$ but rather a significant variation with a $\sim 9$-year periodicity. As we determined the spectrum with a frequency resolution corresponding to $1 / 1320$ cycles/month (del.f $\sim 0.00076$ ), the $11-\mathrm{yr}$ and 9-yr components can be considered well isolated. In addition, from the filtered time series also we can establish 
that the peak separation are 110 months apart rather than 130 months. Often, variations with period between 9 and 13 years are labeled as solar cycle change but the average period in most of the solar activity parameters like sunspot number or solar flux at $10.7 \mathrm{~cm}$. etc turns out to be $\sim 10.5$ years. For example, in his analysis of pulsation activity during 1957 and 1989 as a function of solar wind velocity, Zeiger (1991) used a low pass filter to isolate the solar cycle signal. The only two peaks in $V$ covering the data interval are seen to be 9 years apart (1975 and 1984, as also seen in our Fig. 2(a)), though he identifies it with solar cycle change. The absence of a $\sim 11$-year solar cycle signal in velocity in this interval could be due to the fact that between 1962 and 1973, yearly average speeds did not change much (Gosling et al., 1976) and a clearer signal may emerge when a longer data base would be examined, as is attempted later.

In contrast, $B$ has a $\sim 11$-year cycle with the amplitude in cycle 20 is smaller compared to that seen in cycles 21 or 22 (1976 to 1996). This is in agreement with Slavin et al. (1986) who identified a factor of two enhancement in $B$ between 1976 and 1982 in contrast to the absence of any significant long term change in cycle 20. $B$ variation seems to have an associated 30-year cycle with maximum after the solar maximum of cycle 21 . The periodicity indicated in Fig. 2(b) (second left from bottom) is derived by MEM which is known to provide adequate resolution even for a truncated sinusoid. A similar periodic fluctuation was observed in $A p$ index by Rangarajan and Iyemori (1997). King (1991) had also observed that $B$ during 1978-1984 was greater in magnitude than that for any of the years 1966-1974. We are now able to place this maximum at the peak of a probable longer cycle in $B$. In addition, $B$ has an aperiodic component with a stable part between 1972 and 1980 and an oscillatory component in the latter part. Since the corresponding MEM spectrum did not reveal any significant spectral peak, we are unable to associate any quasi-periodicity with this component. It may be noted that such components could be isolated clearly by SSA whereas techniques like Fourier analysis constrain the components to be sinusoidal. The two minima in this component (third left from bottom in Fig. 2(b)) are separated by nearly 30 years once again. According to King (1979), IMF intensity between 1964 and 1977 showed a significant solar cycle dependence with minimum near solar activity minimum and a broad plateau centered around solar maximum. The initial 10 years of the 10.5 year component shown in Fig. 2(b) matches this description well (though we miss the minimum in 1964 due to paucity of monthly mean values), and by inference, we can expect a slightly different solar-cycle behavior in the next two cycles if the analysis of King (1979) were repeated. Another noteworthy feature in the solar cycle variation in $B$ is the progressive change in the minimum from cycle to cycle. This could be indicative of an underlying longer period component (like the 30-year component extracted and shown in the figure).

A significant fluctuation in $V$ beginning in 1986 and lasting almost until now has an associated 16-month periodicity. Richardson et al. (1994) first drew attention to this significant oscillation. Later, Paularena et al. (1995) showed that these could be detected coincidentally in the $A p$ index and that the only other time when such oscillations were domi- nant in $A p$ was around 1942. In their analysis of $A p$ index, Rangarajan and Iyemori (1997) reconfirmed the results of Paularena et al. (1995) and detected similar but weaker oscillation between 1964 and 1976. The solar wind velocity data presented in Fig. 2(a) does show the weaker signal during the same interval of time. These oscillations in $V$ appear to be also responsible for the analogous periodicity observed in visual aurora by Silverman and Shapiro (1983). It is also significant that their projected minimum of the importance in 1980 coincides quite well with the damped 16-month signal of Fig. 2(a). $B$ varies with a small amplitude range (low percentage contribution) for this quasi-periodic oscillation and when present somewhat significantly, it is noticed during the earlier part between 1967 and 1980 when solar wind velocity did not show this periodicity. This suggests that the solar sources responsible for the signal in $B$ and $V$ may be quite different and some other parameters of the Sun could be examined to see which ones have coherent 16-month oscillation.

Apart from the two major components, others of some significance appear to be a 5-year periodicity and its harmonics ( $~ 30, \sim 20$ months). A 11-month oscillation in the velocity can be related to the types of long-term enhancements identified by Gazis (1996) in the data from 1976 to 1987 between 0.72 and 41.6 AU. The periodicity of these speed enhancements changed between 1 year (reported earlier by Bolton, 1990) to 1.3 years identified by Richardson et al. (1994). Zeiger and Mursula (1998) find that the annual variation in $V$ is more pronounced near solar minima and in successive epochs of minimum it reverses in phase. In Fig. 2(a) we notice that the near-annual signal is clear and uniform from about 1986 whereas earlier it is weaker and mixed with a higher frequency component. Richardson et al. (1994) speculate that the long term speed enhancements may be related to changes in the divergence of the coronal field as suggested by Wang and Sheeley (1991).

A further point of interest in the plots of velocity components (with periods less than the solar cycle) is a fairly consistent change of pattern noticed around and after 1987 . In case of the 42-month periodicity there is a reduction in amplitude while in most other cases one can notice an increase. Around this interval of time, the near annual periodicity changed to 1.3-year cycle also. A cursory examination of the IMF $B$ plots given in Fig. 2(b) shows that a similar change of characteristic in amplitude is centered around 1982. It is possible that the sequence of data gaps in the years 1988, 1990 and 1991 (see Fig. 1) may influence this feature to be an artifact but we believe that the realistic substitution of the missing $V$ values from corresponding $A p$ would ensure the reliability of the results, at least in respect of $V$. A quasi-periodic 60month oscillation and its harmonics (30 and 20 months) are the other significant components in $B$, apart from the solar cycle and 30-year modulations.

A comparison of Figs. 2(a) and 2(b) shows that though some fluctuations are common to both $V$ and $B$, their time profiles are quite different. For example, both the 60- and 30 -month components in $B$ have larger amplitudes in the initial part of the interval under consideration. The 16-month component in $V$ has significant oscillations between 1986 and up until now but in $B$ the amplitudes are relatively large 
from 1967 up to about 1982 suggestive of the fact that the mechanism responsible for enhancement of amplitude in $B$ reduces the amplitude in $V$ and vice versa. The difference in the long term changes in $V$ and $B$ may be due to the inherent differences in their solar sources themselves- $V$ emanating from coronal holes, CMEs and flare-active regions and $B$ from solar magnetic field drawn out by the expanding corona and frozen into it.

The original monthly values, shown in Fig. 1, depict no clearly identifiable long-term variations. SSA enables us to derive individual components by adaptive filtering and to visualize their evolution in time. We know that, of the three cycles, solar cycle 20 was marked by some rather inconsistent pattern in solar wind speed and geomagnetic activity (Gosling et al., 1977; Sontakke et al., 1982) and, therefore, the features reported may not be conclusive. It will be certainly more desirable to identify long term variations in $V$ using some form of proxy data so that the time span can cover several solar cycles. $A p$ and $a a$ indices can eminently suit this purpose. It should be reemphasized that geomagnetic activity is not dependent only on solar wind velocity but is also largely controlled by the southward IMF. Unfortunately, however, one cannot provide an estimate of the IMF intensity directly from any of the known geomagnetic indices.

\subsection{Proxy values of solar wind velocity between 1846} and 1997

Next, we use the monthly mean values of velocity of solar wind between 1965 and 1998 and the corresponding mean monthly values of $A p$ to derive a functional relationship between the two. Only the daily $A p$ values for the days when data for $V$ were also available are taken into consideration to derive the monthly mean values. Maer and Dessler (1964) initially proposed a relationship of the form $V=290 \mathrm{Ap}^{0.22}$. Crooker et al. (1977) found that the six-month average of $A p$ is linearly correlated with the corresponding average of $V$ raised to the power 2.3. They concede that the power curve fitting has the disadvantage of forcing the curve to pass through origin. In other words, when $A p$ tends to zero, the corresponding velocity will also diminish to only zero! Using the three-hour am index, Svalgaard (1977) was able to establish a functional relationship of the form

$$
a m=(0.7 \pm 0.05) V o^{2.25 \pm 0.05}
$$

where $V o$ is $=V / 100$. Once again, this turns out to be a power law curve. According to Gosling et al. (1971), there appears to be a well-established minimum or quiet value of $250-300 \mathrm{~km} / \mathrm{sec}$ in $V$ that does not vary appreciably with solar cycle. Solar wind speed distribution between 1962 and 1974 contained only 13 occasions with velocity in the range of 250 and $275 \mathrm{~km} / \mathrm{sec}$ (Gosling et al., 1976).

For the available monthly mean values of $V$ and $A p$, we attempted to fit a linear relationship in the least squares sense between $\log (V-V t)$ and $\log (A p)$ keeping a threshold of value between 250 to $280 \mathrm{~km} / \mathrm{sec}$ for the velocity $(V t)$ when $A p=0$. We find that the best correlation between observed and calculated velocity is obtained when $V t$ is $250 \mathrm{~km} / \mathrm{sec}$. The scatter plot of the calculated and observed velocities with a correlation coefficient of 0.742 is shown in Fig. 3.

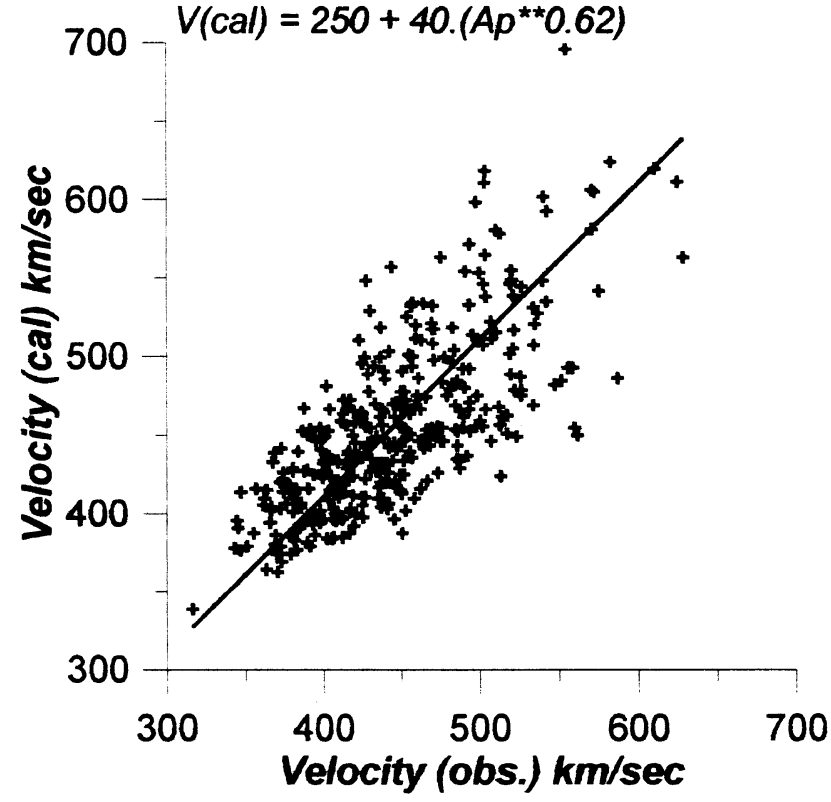

Fig. 3. Scatter plot of the monthly values of $V$ and the corresponding values calculated using the relation between $A p$ and $V$. The correlation between the two is 0.742 .

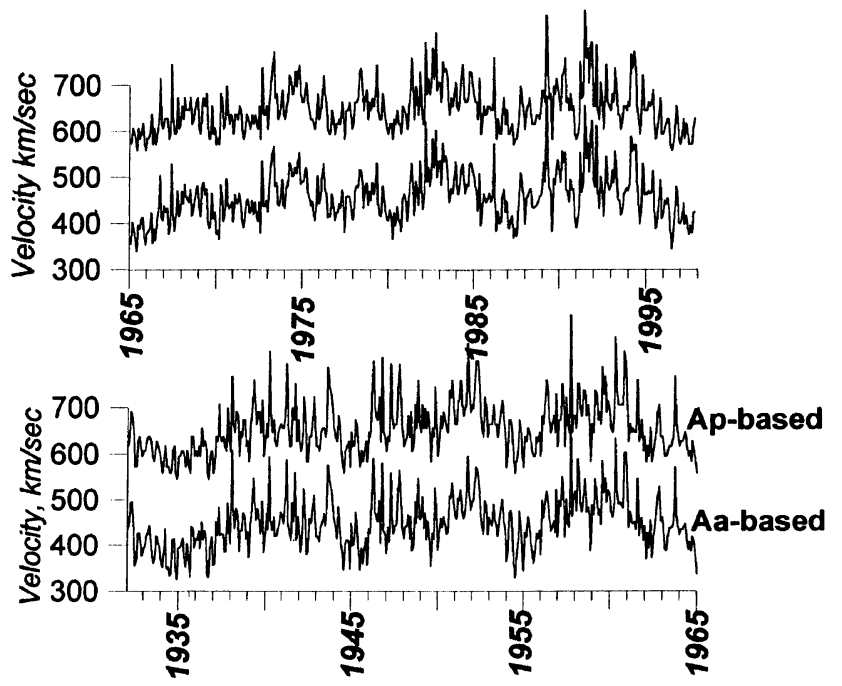

Fig. 4. Monthly values of velocity derived from $A p$ and $a a$ during 1932-1997. $a a$ values are converted to $A p$ values by the relation $(A p(\mathrm{cal})=0.844 a a-5.2)$. Note the shift of $200 \mathrm{~km} / \mathrm{sec}$ of ordinate for $A p$ to avoid overlap.

The calculated velocity is derived from the relation

$$
V \text { cal }=250+40.0\left(A p^{0.62}\right) .
$$

We then computed the linear regression between monthly mean $A p$ and $a a$ values for the interval 1932 to 1998 July to get the relation:

$$
A p(\text { derived })=0.844 a a-5.2
$$

with a correlation coefficient of 0.96 between the two indices. With these two relations, we can now compute a proxy value 


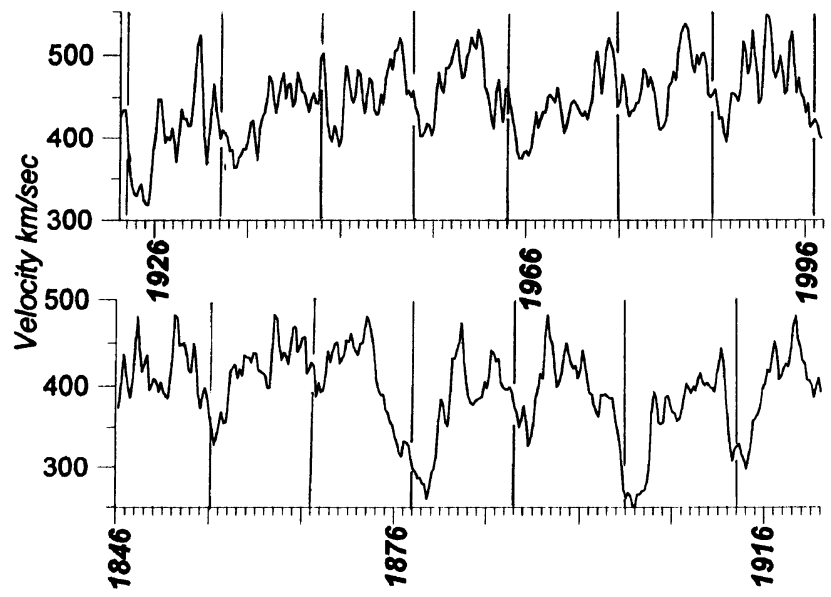

Fig. 5. Monthly values of $V$ derived from $a a$ index and smoothed by 13-month overlapping averages. The vertical lines correspond to epochs of solar minimum.

of solar wind velocity from 1845 using the mean monthly values of $a a$ index between 1845 and 1867 given by Nevanlinna and Kataja (1993) and the published values of $a a$ indices for the latter part. To justify the use of $a a$ indices for derivation of velocity linking it with $A p$ index, we show in Fig. 4 the velocity derived from $A p$ and $a a$ for the period 1932 to 1995. A near-perfect match is immediately apparent. Note that the velocity derived from $A p$ is increased by 200 to avoid overlap. We once again reiterate that geomagnetic activity is not solely due to changes in solar wind velocity and the role of $B z$ is neglected in this derivation of $V$. However, when averaged over longer intervals of time, it is hoped the influence of $B z$ may be minimized, if not eliminated. In Fig. 5 the proxy velocity covering the period 1846 to 1997 are shown. These values are derived as 13-month overlapping averages of the monthly values of $V$. The solar minimum epochs are marked by vertical lines. The velocity variations in individual solar cycles appear to be somewhat well-ordered with minimum velocity coinciding with or just after the solar minimum and a maximum somewhere in the middle of the solar cycle.

Based on the time variations of annual mean $a a$ index, Feynman and Crooker (1978) estimated that around 1900 the average solar wind speed was as much as a factor of two lower or the southward component of IMF as much as a factor of 3 smaller or both than at present. According to them, a longer period ( $\sim 80$ year cycle) variation had its minimum around the year 1900. It can be seen from Fig. 5 that the derived velocity reached its lowest imposed value of $250 \mathrm{~km} / \mathrm{sec}$ (with $a a$ index being negative for some months in 1901 and 1902) whereas the minimum value in cycle 22 was around $400 \mathrm{~km} / \mathrm{sec}$. The possibility of 1900 being the minimum in the longer period oscillation is further borne out by the fact there is, in general, an increase in the minimum value of velocity if we move backwards in time up to at least 1846. Feynman (1982) showed that the rise in amplitude of aa was well ordered from 1900 to 1960 but prior to 1900 it was not so. The proxy velocity, on the other hand, clearly defines the minimum in 1900 with increase on either side. However, one does not see the tendency of any decline in the minimum velocity after 1966 but a somewhat complex pattern.

The reconstructed components constituting the velocity variations between 1846 and 1997 are shown in Figs. 6(a) and 6(b). In contrast to the earlier results for the in-situ measurements, almost 90 percent of the total variability in velocity could be explained by the first four components and the balance by few other quasi-periodic fluctuations shown in the figure. The most dominant (45 percent variability accounted for) is the long term trend discussed earlier. One can immediately see a pattern in this trend with a smooth rise between 1902 and 1958 and a decline marked with a quasi-periodic oscillation in the initial part. The second most significant component is the solar cycle component (with a nominal 10.8 year periodicity) and its two harmonics. The largest amplitudes of the solar cycle change in velocity are seen between 1870 and 1914. A peculiar signature of the solar cycle is seen beginning with cycle 20 (1964) unlike any other epoch with the velocity showing a steady rise to the peak much later in the sunspot cycle compared to other cycles. This odd behavior of the solar wind speed was commented upon by Gosling et al. (1977) who found that high speed streams and streams with velocity in excess of 700 $\mathrm{km} / \mathrm{sec}$ were far more common in 1973-75 than near solar maximum. On the other hand, the sunspot series did not differ significantly from the previous cycles.

A 22-year cycle (also called the Hale cycle) in geomagnetic activity has been identified and it was attributed by Russell and McPherron (1973) to the fact that while the solar wind is ordered in the solar-equatorial coordinate (GSEQ) system, its interaction with the magnetosphere is ordered in the solar-magnetosphere (GSM) system. Cliver et al. (1996), however, argue that the intrinsic solar variation other than polarity reversal in the systematic low-high alternation of even-odd sunspot maxima is the dominant cause of this signal in geomagnetic activity. They also add that various lines of evidence indicate that the 22-year cycle of geomagnetic activity has been a quasi-regular feature over 150 years.

Figure 6 clearly reveals that in the solar wind speed, derived using geomagnetic index $a a$, does not have the double solar cycle component embedded in it. One possible reason could be the fact the Hale cycle manifests as enhanced transient activity during the rise and maximum of odd cycles and as 27-day recurrence during the declining part of the even cycles (Cliver et al., 1996) and the two separate components may tend to mask the signal. It is also possible that in a a index, while the Hale cycle does exist, its amplitude does not exceed an adequate threshold minimum level to manifest itself in the derived velocity component.

To examine the relationship of 11-year solar wind component with the sunspot cycle in detail, we show in Fig. 7 the annual mean sunspot number $(R z)$ as also the first principal component derived by SSA of $R z$ covering the same period of time. Result of cross-correlation between the two $\sim 11$-year oscillations is depicted at the top in Fig. 7 . The correlation pattern clearly establishes the persistence of the solar control of the 11-year oscillation throughout the period of analysis. The maximum correlation is obtained at a lag of 22 months, in the declining phase of the solar cycle. In other words, velocity maximum takes place with a lag of nearly 


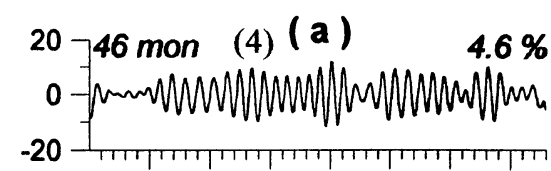

(3)

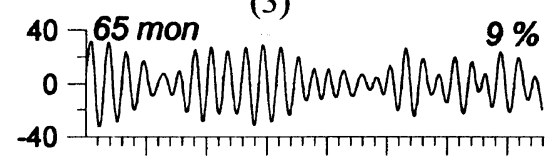

(2)
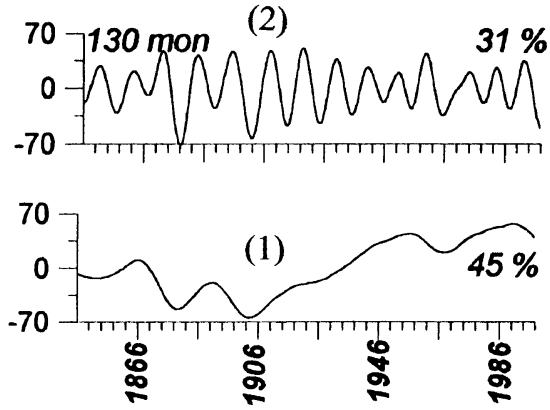

(b)

(8)
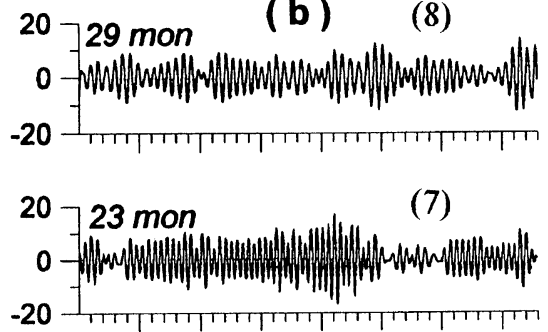

(6)

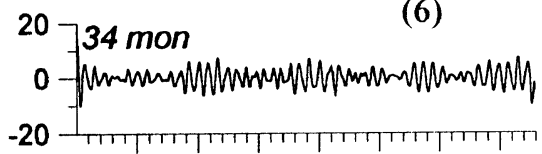

(5)

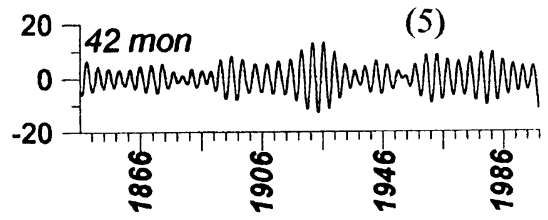

Fig. 6. The reconstructed components of $V$ derived by SSA applied to the proxy value series.

Table 1.

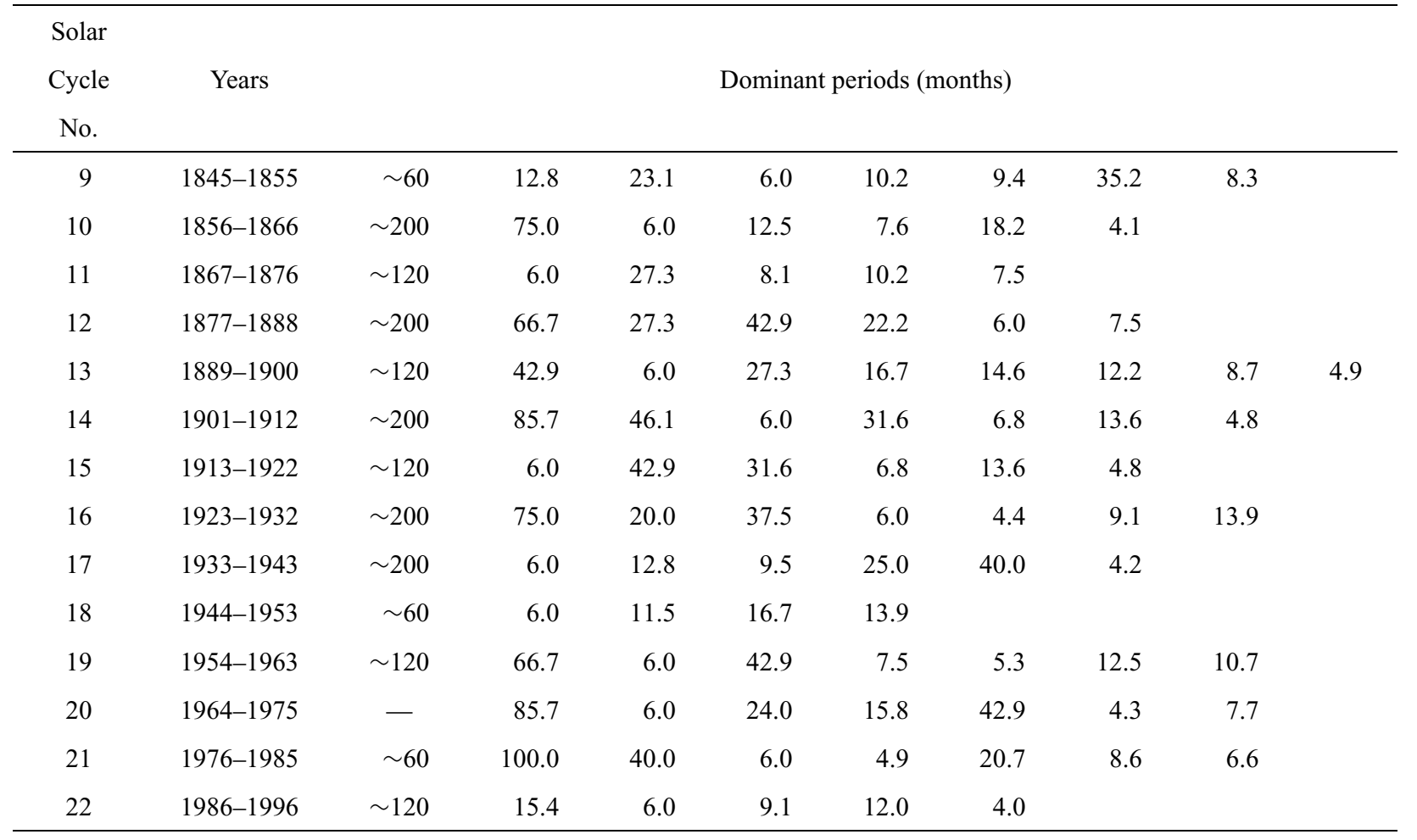

Note: The dominant periods are listed in decreasing order of the relative contribution to the total variance of the time series for each group of years.

two years after the sunspot has attained its peak value in each cycle. Another noteworthy aspect of the $V$ variation is the fact that the amplitude does not bear any relation to that of the 11-year component in $R z$. The peculiar signature in $V$ seen in cycle 20 is absent in $R z$ and least amplitude in $R z$ during cycle 12 are marked by large signals in $V$. Feynman (1982) separated $a a$ index into two parts, one dependent on sunspot and the other independent of it, with both parts having nearly equal amplitude. Both exhibit solar cycle variation but with different phases. The present result implies that the main cause of the solar cycle variation in solar wind velocity is the recurrent high speed solar wind streams, most common in the declining part of each solar cycle, while the sunspotdependent part of $V$ does not appear to be very well-ordered. This is in conformity with Feynman (1982) who found well marked maxima in yearly averages of $V$ during the declining 

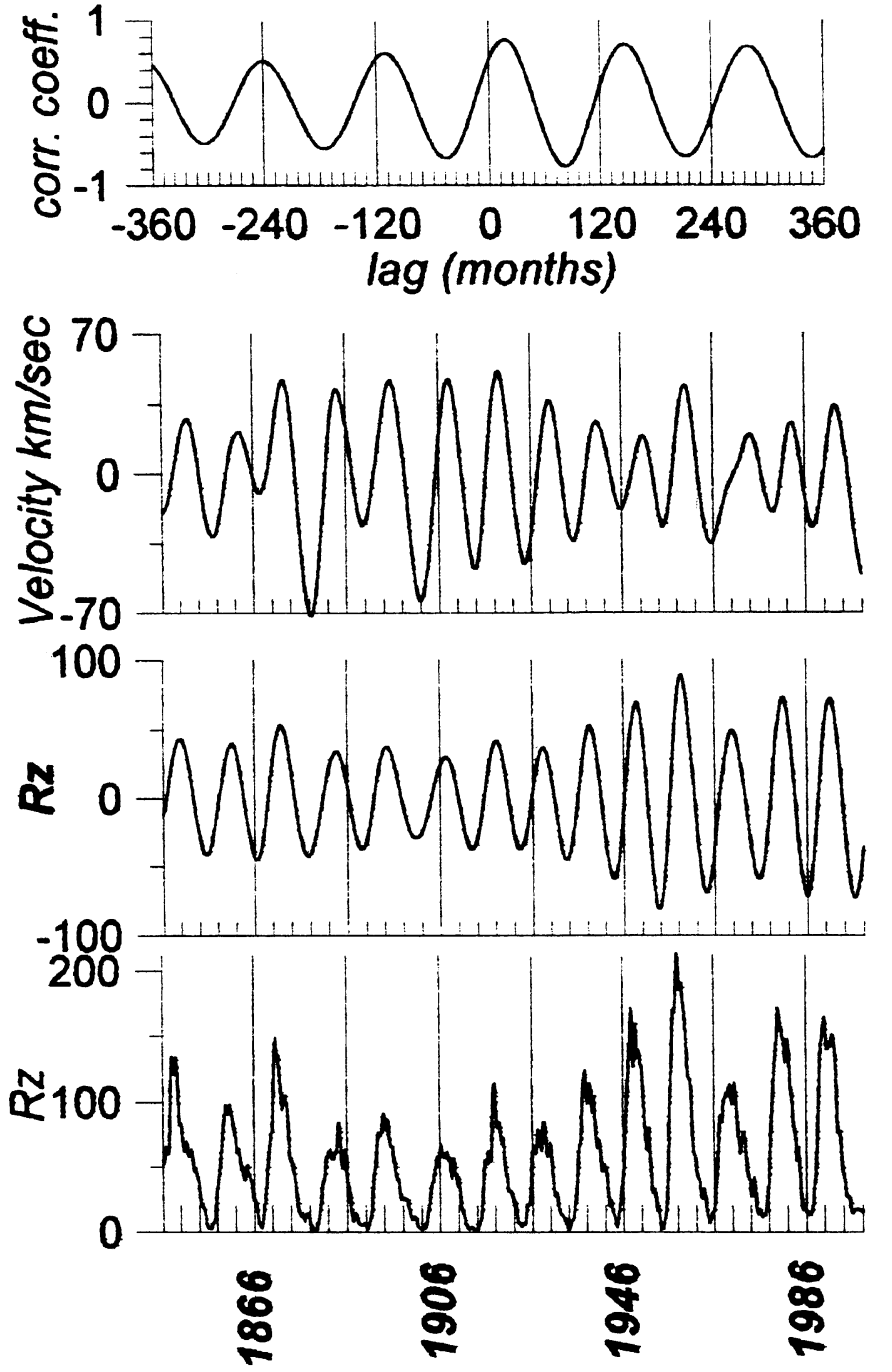

Fig. 7. Solar cycle component in $V$ compared with the same in sunspot number $(R z)$. At the bottom are the annual values of $R z$. At the top is the cross correlation between $V$ and $R z$ (11-year component) as a function of lag in months. Positive lags imply delay in the appearance of peak in $V$ relative to $R z$.

phase and a minimum one year after solar minimum. Coronal Mass Ejections (CME) are relatively frequent during solar maximum epochs and can contribute significant mass to the near-ecliptic solar wind (Webb and Howard, 1994) but apparently the CMEs do not contribute in an equal measure to any systematic enhancement of solar wind speed.

About 7 percent of the variability in the long series of solar wind speed are contributed by the components depicted in Fig. 6(b) (in decreasing order of importance). Of these, a variation with $\sim 42$ month periodicity may be considered significant because a nearly analogous periodicity was detected in the equatorial Dst index by Rangarajan and Araki (1997) and in $A p$ index by Rangarajan and Iyemori (1997) who attributed the quasi-periodic fluctuations in geomagnetic activity to the change in solar wind speed with the passage of IMF sector boundary past the earth. Indeed, the sector structure of IMF exhibited a $\sim 3.7$ year periodic variation (Gonzalez and Gonzalez, 1987). The significance or otherwise of the other three components could not be easily established.
As mentioned earlier, a perceptible difference in solar wind speed and geomagnetic activity was observed between cycles 20 and 21 or 22 . It is likely that other cycles between 9 and 22 may also show individual peculiarities in terms of the variations in $V$. To evaluate this, the derived monthly values of $V$ between 1845 and 1996 are subjected to SSA again, now restricting the data to cover individual solar cycles between cycles 9 and 22. Dominant periodicities detected from the principal components through maximum entropy spectral analysis of the monthly values are listed in Table 1. The listing is done in the descending order of significance of the spectral components. This table clearly shows that while the broad pattern of velocity maximum following solar maximum and least velocity at or following solar minimum is preserved in all the cycles, individual cycles show that the longer period variations are highly variable (between 120 and 200 months). Periods close to half a solar cycle is seen in most cases but only the semiannual oscillation appears as common in all the cycles. The lack of consistency may be attributed largely to the influence of sporadic bursts in the geomagnetic activity enhancing some monthly values arbitrarily and consequently the derived velocity too.

\subsection{Statistical relation between $A p$ index and solar wind parameters}

In this section, we examine the nature of relationships existing between solar wind and IMF parameters and geomagnetic activity, represented by $A p$ index. For this purpose we use the daily averages of $A p$ and in-situ observations of the parameters covering the period 1964 to 1998 July.

It may be mentioned that similar and more elaborate exercises have been carried out in the past but with limited data sets (for e.g., Maezawa, 1978). Results derived here confirm that most of the results established earlier are stable over at least the past three solar cycles. The IMF and solar wind parameters are divided into 11 (or 10) different bins with increasing magnitudes and the averages are computed for each bin as also the corresponding daily mean Ap values. Change in $A p$ with increase in $V, B$ and $N$ (density of solar wind) are shown in Figs. 8(a), (b) and (c). Each panel depicts the changes during four intervals of time: covering the entire period 1964-1998 and each of the three solar cycles 20, 21 and 22 . This enables us to bring out whether the observed dependence of geomagnetic activity on $V, B$ and $N$ is consistent throughout or not.

Fairly systematic increase in $A p$ in association with change in $V$ can be seen for all the solar cycles. Ap magnitudes, over a moderate range, during cycle 20 and 22 depict similar dependence on $V$ while cycle 21 covers a much larger range with a distinctly sharper increase with solar wind velocity in excess of about $700 \mathrm{~km} / \mathrm{sec}$ which may be due to higher speed streams emanating from CMEs and flare-active regions, which are known to be responsible for severe, sporadic geomagnetic storms.

The pattern associating change in $A p$ magnitude with increasing IMF $B$, on the other hand, is practically similar over all the three solar cycles with a near linear increase in the magnitude of $A p$ with enhanced $B$. Contrary to the observed peculiarities of solar wind during cycle 20 pointed out by Gosling et al. (1977), we note that the solar wind control of geomagnetic activity, through both $V$ and $B$, during cycle 

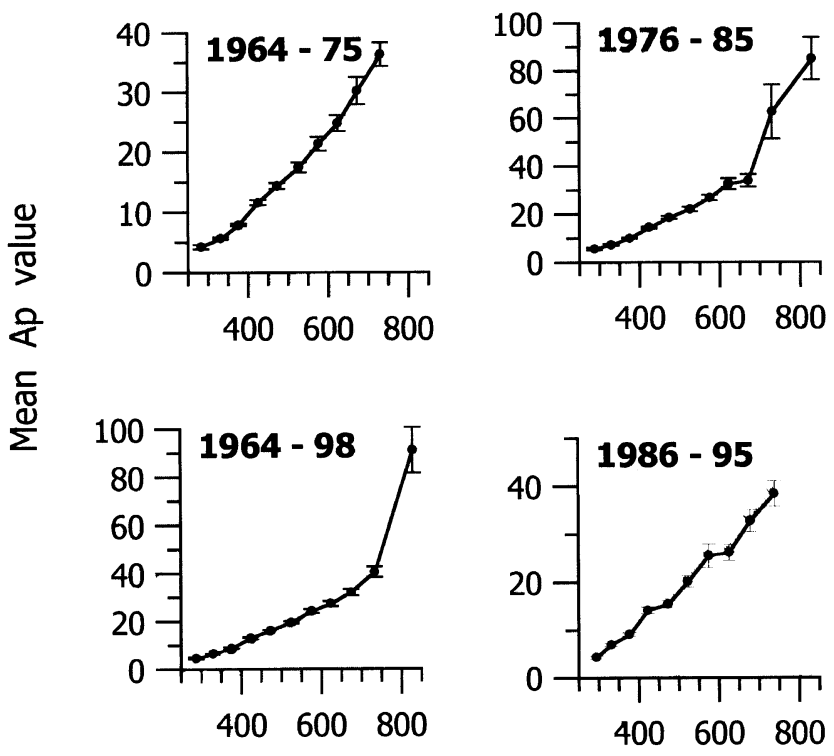

Velocity $\mathrm{km} / \mathrm{sec}$

Fig. 8(a). Statistical relation between $A p$ arranged in 12 categories of increasing level of geomangetic activity and corresponding averages of solar wind velocity. Daily values are used. The four panels correspond to the results of analysis separately for three solar cycles (20,21 and 22) and for the entire interval 1965-1998.
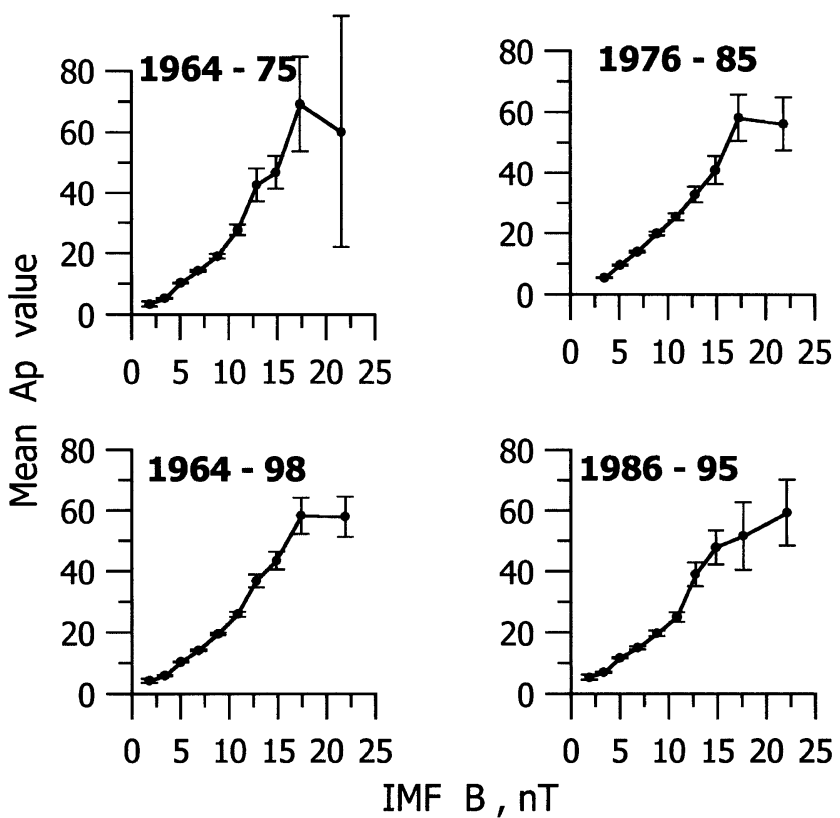

Fig. 8(b). Same as Fig. 8(a), but for IMF intensity ( $B)$.

20 is very similar to that noticed for cycle 22 and for the entire interval. $A p$ dependence on solar wind density appears rather insignificant with no commonality of the patterns for the three solar cycles. Let us recall that, according to King (1991), density prior to and after 1971 may not be directly comparable. Differences in normalization for instruments and observations apparently have no appreciable effect, as seen from a comparison for three solar cycles. In all the four panels, the magnitude of $A p$ does not exceed 20 if we ignore

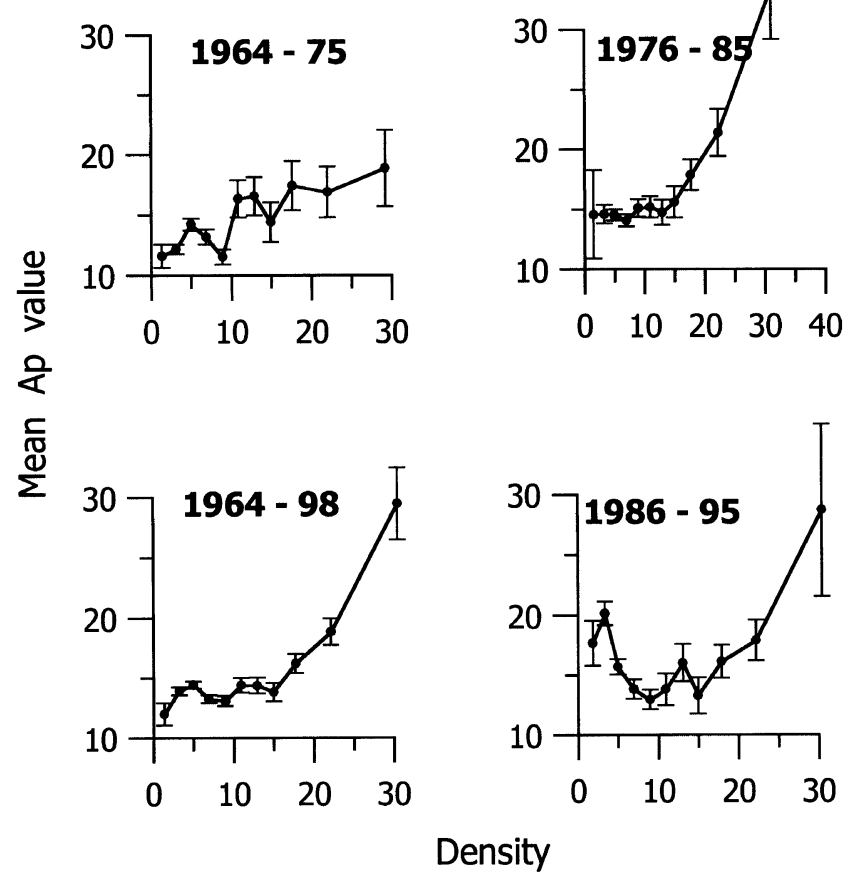

Fig. 8(c). Same as Fig. 8(a) but for plasma density $(N)$.

the last bin with fewer data points, suggestive of the lack of direct link between solar wind density and geomagnetic activity.

We next consider the changes in sixteen solar wind and IMF parameters and their variability and their suitable combinations, as outlined in literature. The variables utilized are listed below:

$V, B, N, B x, B y e, B z e, B y m, B z m$, sig. $V$, sig. $B$, sig. $N$, sig. $B x$, sig. $B y e$, sig. $B z e$, sig. $B y m$, sig. $B z m, V o^{2}, V o^{2.25}$, $V o^{2} B z m, V o^{2} B z e, V o^{2} B s m, V . B s e$ and $V . B s m$

sig. denotes the standard deviation of the parameter (as given in the OMNI tape), Vo stands for $V / 100$ (as used by Svalgaard, 1977); $E$ and $M$ correspond to whether the components are derived in the GSE or GSM coordinate systems.

The choice of the combinations of $V$ and $B$ is motivated by the publications of Crooker et al. (1977) who find $V^{2}$ to be correlated with $A p$, of Svalgaard (1977) who associated $V^{2.25}$ with three hourly am index, of Murayama and Hakamada (1975) and Crooker and Gringauz (1993) who link $B s V^{2}$ with geomagnetic activity and of Smart et al. (1979) who correlate $V . B z m$ and $V^{2}$.Bzm with $A E, A p$ and Dst and of Maezawa (1978) who find the effect of $B, V$ and the latitude of IMF to be multiplicative.

In contrast to the limited data that was available when the listed works were carried out, we now have data covering three solar cycles. Third degree orthogonal polynomials are fitted to the data and the equations of the form:

$$
Y=A o+A 1 \cdot X+A 2 \cdot X^{2}+A 3 \cdot X^{3}
$$

where $X$ represents any of the 23 solar wind/IMF parameters or their combinations and $Y$ represents $A p$ values. 11 (or 10) pairs of $X$ and $Y$ are used to find the coefficients $A o, A 1, A 2$ and $A 3$. These are listed in Table 2. 
Table 2.

\begin{tabular}{|c|c|c|c|c|}
\hline Parameter & Ao & $A 1$ & $A 2$ & $A 3$ \\
\hline$V$ & $-0.21882725 \mathrm{E}+02$ & $0.12955633 \mathrm{E}+00$ & $-0.18524842 \mathrm{E}-03$ & $0.16842320 \mathrm{E}-06$ \\
\hline$B$ & $0.88997506 \mathrm{E}+01$ & $-0.28286213 \mathrm{E}+01$ & $0.61414374 \mathrm{E}+00$ & $-0.17375196 \mathrm{E}-01$ \\
\hline$B x$ & $0.12306797 \mathrm{E}+02$ & $0.52055725 \mathrm{E}-01$ & $0.23794574 \mathrm{E}+00$ & $-0.41238825 \mathrm{E}-02$ \\
\hline Bye & $0.10840750 \mathrm{E}+02$ & $-0.73293338 \mathrm{E}-01$ & $0.28602991 \mathrm{E}+00$ & $0.14926360 \mathrm{E}-02$ \\
\hline Bze & $0.13956313 \mathrm{E}+02$ & $-0.11391092 \mathrm{E}+01$ & $0.54361937 \mathrm{E}+00$ & $-0.32431430 \mathrm{E}-01$ \\
\hline Bym & $0.12356934 \mathrm{E}+02$ & $0.55136731 \mathrm{E}+00$ & $0.21877134 \mathrm{E}+00$ & $-0.98874473 \mathrm{E}-02$ \\
\hline Bzm & $0.11696814 \mathrm{E}+02$ & $-0.42372121 \mathrm{E}+01$ & $0.58970466 \mathrm{E}+00$ & $0.20427725 \mathrm{E}-01$ \\
\hline $\operatorname{sig} . V$ & $0.25111065 \mathrm{E}+01$ & $0.95477955 \mathrm{E}+00$ & $-0.23621526 \mathrm{E}-01$ & $0.25618844 \mathrm{E}-03$ \\
\hline $\operatorname{sig} . B$ & $0.14181162 \mathrm{E}+01$ & $0.91389418 \mathrm{E}+01$ & $-0.55665160 \mathrm{E}+00$ & $0.30870518 \mathrm{E}-01$ \\
\hline sig. $B x$ & $0.13179509 \mathrm{E}+02$ & $-0.59278970 \mathrm{E}+01$ & $0.28165148 \mathrm{E}+01$ & $-0.16763645 \mathrm{E}+00$ \\
\hline sig. Bye & $0.73968102 \mathrm{E}+01$ & $0.20040374 \mathrm{E}+00$ & $0.10427041 \mathrm{E}+01$ & $-0.63908804 \mathrm{E}-01$ \\
\hline sig. Bze & $0.10885549 \mathrm{E}+02$ & $-0.31009811 \mathrm{E}+01$ & $0.17851471 \mathrm{E}+01$ & $-0.93175359 \mathrm{E}-01$ \\
\hline sig. Bym & $0.72997917 \mathrm{E}+01$ & $0.24424173 \mathrm{E}+00$ & $0.10405916 \mathrm{E}+01$ & $-0.64218819 \mathrm{E}-01$ \\
\hline sig. $B z m$ & $0.84734326 \mathrm{E}+01$ & $-0.88721445 \mathrm{E}+00$ & $0.12751508 \mathrm{E}+01$ & $-0.62257716 \mathrm{E}-01$ \\
\hline$V o^{* * 2}$ & $-0.14451772 \mathrm{E}+02$ & $0.25549152 \mathrm{E}+01$ & $-0.68364869 \mathrm{E}-01$ & $0.75659972 \mathrm{E}-03$ \\
\hline$V o^{* * 2.25}$ & $-0.12901586 \mathrm{E}+01$ & $0.54999962 \mathrm{E}+00$ & $-0.56564398 \mathrm{E}-03$ & $-0.10273714 \mathrm{E}-04$ \\
\hline$V o^{* * 2 . B z e}$ & $0.14004798 \mathrm{E}+02$ & $-0.12584380 \mathrm{E}+00$ & $0.23082783 \mathrm{E}-02$ & $0.52744605 \mathrm{E}-05$ \\
\hline$V o^{* * 2 . B z m}$ & $0.14873127 \mathrm{E}+02$ & $-0.11297565 \mathrm{E}+00$ & $0.12450724 \mathrm{E}-02$ & $-0.39879619 \mathrm{E}-05$ \\
\hline V.Bse & $0.78307688 \mathrm{E}+01$ & $0.21939590 \mathrm{E}-01$ & $-0.61924923 \mathrm{E}-05$ & $0.16301165 \mathrm{E}-08$ \\
\hline V.Bsm & $0.86177222 \mathrm{E}+01$ & $0.17140989 \mathrm{E}-01$ & $-0.26186296 \mathrm{E}-05$ & $0.12256441 \mathrm{E}-08$ \\
\hline
\end{tabular}

Coefficients of the third degree polynomial of the form

$$
Y=A o+A 1 * X+A 2 * X * * 2+A 3 * X * * 3
$$

where $X$ is any one of the listed IMF/solar wind parameters and $Y$ the corresponding $A p$ values. Note that $V o=V / 100$.

Though in some cases a third degree equation was found redundant from the consideration of residual variance, we provide the constants for all the four coefficients. From the exponent and the nature of the graphs governing the relationship, linearity or otherwise can be established easily.

Plots of the IMF/solar wind parameters and corresponding $A p$ are shown in Fig. 9. Some of the figures left out are similar to the others of the same type. For example, sig.Bze and sig.Bzm or sig.Bye and Sig.Bym are practically identical and are shown as sig. $B z$ and sig. $B y$ respectively.

The parameters sig. $B$, sig. $B x$, sig. $B z, V . B s e, V . B s m$, $V o^{2.25}$ all are very nearly linearly related to $A p$. In a similar fashion, the parameters $B z e, B z m$ and $V o^{2.25} B s$ appear to have inverse linear relation. This should only to be expected as the negative $B z$, corresponding to southward directed IMF, is the crucial parameter in solar wind-magnetosphere interaction and enhanced southward field tends to be responsible for greater geomagnetic activity.

On the other hand, $V o$ and $V o^{2}$ show two different segments of linear relationship; larger change in the controlling parameter causing somewhat less change in the initial transition from quiet to a disturbed state and more rapid change in the disturbed conditions for a smaller range of change in the velocity or its squared value. Plasma density and its variability exhibits a complex relation unlike any of the other parameters.

Some of the other aspects which appear to be significant are the following:

For both $B x$ and $B y, A p$ dependence is nearly symmetric with respect to the zero value of the IMF component, indicative of the fact that there is no preferential interaction between the solar wind and magnetosphere for one type of polarity or the azimuthal direction over the other.

In the plots of $B z$ vs. $A p$, the segment when $B z$ is northward does not really correspond to values of $A p$ close to zero but nearer 10 on the average.

IMF $B$ has an average magnitude of about 4 when the geomagnetic activity is at quiet levels $(A p<=4)$.

Variability in the components of IMF $B$ tend to be very small (close to zero) when geomagnetic calm prevails with $(A p<10)$.

Thus we may infer that when the magnetosphere is in a state of extreme calm, the interplanetary parameters can be assumed to have the following "Quiet State" values:

$$
\text { Velocity } \sim 335 \mathrm{~km} / \mathrm{sec}
$$



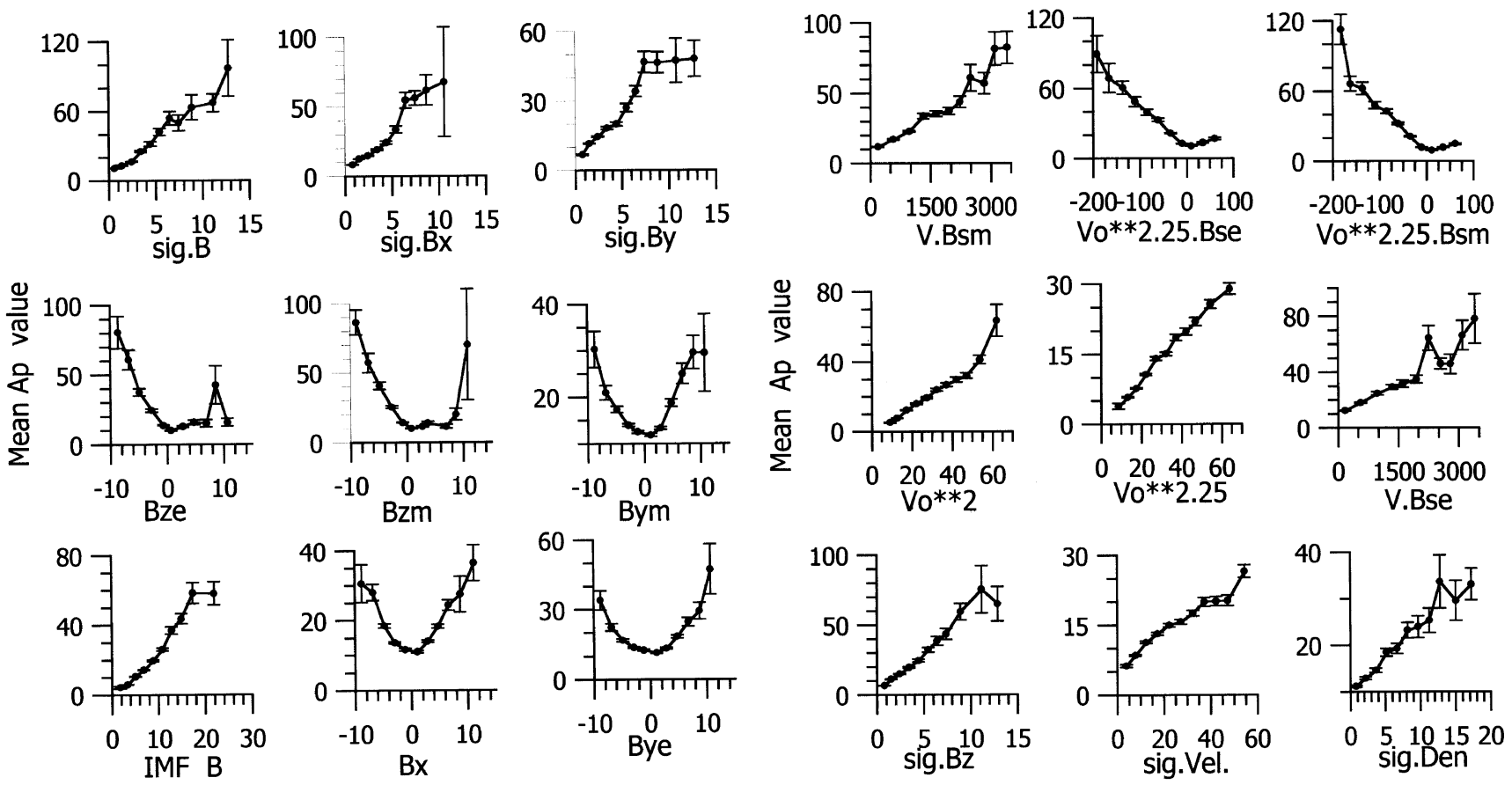

Fig. 9. Statistical relation between average $A p$ values in 12 categories of increasing level of geomagnetic activity and the corresponding averages of several solar wind/IMF parameters and their combinations. The equations relating the solar wind parameters with $A p$ for each panel are given in Table 2 .

\author{
Bsm or Bse 0 \\ $B z$ (northward) $<1 \mathrm{nT}$
}

IMF $B \sim 4 \mathrm{nT}$

Variability in IMF $B$ components $\sim 1$ to $1.5 \mathrm{nT}$.

Gussenhoven (1988) suggested that interplanetary conditions with $B<5 \mathrm{nT},|B z|<2 \mathrm{nT}$ and $V<400 \mathrm{~km} / \mathrm{sec}$ for defining a baseline magnetosphere in which coupling with solar wind is minimal. Kerns and Gussenhoven (1990) identified baseline magnetosphere with prolonged periods of low values of geomagnetic activity indices $a m, K p$ or $A E$ and the resulting baseline conditions were given as $V<=390$ $\mathrm{km} / \mathrm{sec}, B<=6.5 \mathrm{nT}$ and persistence of the conditions for at least 5 hours. Our threshold values, particularly for velocity is significantly lower.

One can use either the magnitude of geomagnetic activity or the causative solar wind parameter(s) and the equations of Table 2 to estimate either the probable state of the interplanetary medium or that of the magnetosphere.

\section{Conclusions}

Long term variations in solar wind velocity and IMF intensity are examined using first the spacecraft observations covering three solar cycles and later using the monthly values of $a a$ index as a possible indicator of the speed of solar wind. While the in-situ data set fails to reveal a clear solar cycle signal in velocity but rather the presence of a $\sim 9$-year cycle, the solar cycle signal in $V$ is clearly brought out in the proxy data. The speed maximizes about 22 months after the solar maximum and the minimum occurs a year later than solar minimum. No relation exists between the amplitude of the 11-year signal in $R z$ and that in $V$. Individual cycles between 9 and 22, however, do not share any common pe- riodicity except the semiannual variation. IMF $B$, between 1965 and 1998, clearly manifests the solar cycle component with variable amplitude. A secular increase in the minimum of this component is also indicated. Some significant quasiperiodic fluctuations found in solar wind speed such as the $\sim 44$ mon. and $\sim 16$ mon. oscillations apparently lead to similar changes in geomagnetic activity.

In the statistical study linking $A p$ and different solar wind parameters, we find that the relationship between $V, B$ and $N$ with $A p$ appears quite stable over three solar cycles, though cycle 21 exhibits somewhat different behavior, particularly under disturbed conditions. Bieber et al. (1993) find that the association between sunspot cycle and low frequency IMF turbulence waves was more pronounced in cycle 21 compared to cycle 20. Perhaps, these features are linked in some manner. When the magnetosphere is somewhat less disturbed, large change in velocity leads to less significant changes in geomagnetic activity but after a level of disturbance, corresponding to $A p \sim 25$, even smaller increase in wind speed can cause significant change in the geomagnetic activity levels. In contrast, the association between IMF $B$ and $A p$ appears almost linear over a considerably wider range of geomagnetic activity from the base level $(A p \sim 0)$ to the level with $A p \sim 60$.

Solar wind electric field, represented by $V . B s$ is linearly correlated with $A p$. Similar linear relationship is also seen for the variability in the components of $B$. From the statistical relations, we are able to estimate a base level for the interplanetary conditions during which the magnetosphere is expected to be quiescent.

Acknowledgments. This work would not have been possible but for the excellent cooperation extended by Prof. Iyemroi of World Data Center for Geomagnetism and Space Magnetism, Kyoto Uni- 
versity, Kyoto Japan who extracted the daily averages of solar wind parameters from the OMNI tape for us and offered suggestions on the use of the data. Our grateful thanks to him. We also express our thanks to one of the referees whose elaborate and constructive criticisms have helped in considerable improvement in the quality of the presentation. GKR would like to express his gratitude to $\mathrm{CNPq}$, Brazil for the offer of a fellowship to work as Visiting Professor at Observatorio Nacional and to the Director of Observatorio Nacional for the facilities provided

\section{References}

Bieber, J. W., J. Chen, W. H. Mathaeus, C. W. Smith, and M. A. Pomerantz, Long term variations of interplanetary magnetic field spectra with implications for cosmic ray modulation, J. Geophys. Res., 98, 3585-3603, 1993.

Bolton, S. J., One year variations in the near earth solar wind, Geophys. Res. Lett., 17, 37-40, 1990.

Bruno, R., U. Villante, and A. Stecca, Selected solar wind parameters at 1 AU through two solar activity cycles, Ann. Geophys., 12, 105-112, 1994.

Cliver, E. W., V. Boriakoff, and K. H. Bounar, The 22-year cycle of geomagnetic and solar wind activity, J. Geophys. Res., 101, 27094-27109, 1996.

Crooker, N. U. and K. I. Gringauz, On the low correlation between longterm averages of solar wind speed and geomagnetic activity after 1976, J. Geophys. Res., 98, 59-62, 1993.

Crooker, N. U., J. Feynman, and J. T. Gosling, On the high correlation between long-term averages of solar wind velocity and geomagnetic activity, J. Geophys. Res., 82, 1933-1937, 1977.

Dettinger, M. D., M. Ghil, C. M. Strong, W. Weibel, and P. Yiou, Software expedites singular spectrum analysis of noisy time series, EOS Trans. $A G U, \mathbf{7 6}(2), 12,14,21,1995$.

Feldman, W. C., J. R. Asbridge, and S. J. Bame, Long term variations of selected solar wind properties, IMP 6, 7 and 8 results, J. Geophys. Res., 83, 2177-2189, 1978.

Feynman, J., Geomagnetic and solar wind cycles 1900-1975, J. Geophys. Res., 87, 6133-6162, 1982.

Feynman, J., Solar cycle and long term changes in the solar wind speed, Rev. Geophys. Space Phys., 21, 338-348, 1983.

Feynman, J. and N. U. Crooker, The solar wind at the turn of the century, Nature, 275, 626-627, 1978.

Gazis, P. R., Long term enhancements in the solar wind speed, J. Geophys. Res., 101, 415-424, 1996.

Gazis, P. R., J. D. Richardson, and K. I. Paularena, Long term periodicity in solar wind velocity during the last three solar cycles, Geophys. Res. Lett., 22, 1165-1168, 1995.

Gonzalez, A. L. C. and W. D. Gonzalez, Periodicities in the interplanetary magnetic field polarity, J. Geophys. Res., 92, 4357-4375, 1987.

Gosling, J. T., R. T. Hansen, and S. J. Bame, Solar wind speed distributions: 1962-1970, J. Geophys. Res., 76, 1811-1815, 1971.

Gosling, J. T., J. R. Asbridge, and W. C. Feldman, Solar wind speed variations: 1962-1974, J. Geophys. Res., 81, 5061-5070, 1976.

Gosling, J. T., J. R. Asbridge, and S. J. Bame, An unusual aspect of solar wind speed variation during solar cycle 20, J. Geophys. Res., 82, 33113314, 1977.

Gussenhoven, M. S., Low altitude convection, precipitation and current patterns in the baseline magnetosphere, Rev. Geophys. Space Phys., 26, 792-808, 1988.

Kerns, K. J. and M. S. Gussenhoven, Solar wind conditions for a quiet magnetosphere, J. Geophys. Res., 95, 20869-20875, 1990.

King, J. H., Solar cycle variations in IMF intensity, J. Geophys. Res., 84, 5938-5949, 1979

King, J. H., Long-term solar wind variations and associated data sources, $J$. Geomag. Geoelectr., 43 Suppl., 865-880, 1991.

Maer, K. and A. J. Dessler, Comments on the paper by Conway W. Snyder et al., J. Geophys. Res., 69, 2846, 1964.

Maezawa, K., Dependence of geomagnetic activity on solar wind parameters, a statistical approach, Solar Terr. Environ. Res. Japan, 2, 103-121, 1978 .
Mayaud, P. N., Derivation, Meaning and use of geomagnetic indices, 154 pp., Geophys. Monograph 22, AGU, Washington D.C., 1980.

Murayama, T. and K. Hakamada, Effect of solar wind on the development of magnetospheric substorms, Planet. Space Sci., 23, 75-91, 1975.

Nevanlinna, H. and K. Kataja, Na extension of geomagnetic activity index a a for two solar cycles (1844-1867), Geophys. Res. Lett., 20, 2703-2706, 1993.

Paularena, K. I., A. Szabo, and J. D. Richardson, Coincident 1.3-year in the ap geomagnetic index and the solar wind, Geophys. Res. Lett., 22, 3001-3004, 1995

Penland, C., M. Ghil, and K. M. Weickmann, Adaptive filtering and maximum entropy spectra with applications to changes in atmospheric angular momentum, J. Geophys. Res., 96, 22659-22671, 1991.

Rangarajan, G. K., Sunspot variability and an attempt to predict cycle 23 by adaptive filtering, Earth Planets Space, 50, 91-100, 1998.

Rangarajan, G. K. and T. Araki, Mutiple time scales in the fluctuations of equatorial Dst index through singular spectrum analysis, J. Geomag. Geoelectr., 49, 3-20, 1997.

Rangarajan, G. K. and T. Iyemori, Time variations of geomagnetic activity indices $K p$ and Ap: an update, Ann. Geophys., 15, 1271-1290, 1997.

Richardson, J. D., K. I. Paularena, J. W. Belcher, and A. J. Lazarus, Solar wind oscillations with a 1.3-year period, Geophys. Res. Lett., 21, 15591560, 1994.

Rickett, B. J. and W. A. Coles, Evolution of the solar wind structure over a solar cycle-interplanetary scintillation velocity measurements compared with coronal observations, J. Geophys. Res., 96, 1717-1736, 1991.

Russell, C. T. and R. M. McPherron, Semiannual variation in geomagnetic activity, J. Geophys. Res., 78, 92-108, 1973.

Silverman, S. M., Magnetic activity at and preceding solar minimum for the past 500 years, J. Geophys. Res., 91, 10157-10161, 1986.

Silverman, S. M. and R. Shapiro, Power spectral analysis of auroral occurrence frequency, J. Geophys. Res., 88, 6310-6316, 1983.

Slavin, J. A., G. Jungman, and E. J. Smith, The interplanetary magnetic field observations during solar cycle 21: ISEE 3/ICE observations, Geophys. Res. Lett., 13, 513-516, 1986.

Smart, D. F., H. B. Garrett, and M. A. Shea, The prediction of $A E$, ap and Dst at time lags between 0 and 30 hours, in Solar Terrestrial Prediction Proceedings vol. 2, edited by R. F. Donnelly, 733 pp., NOAA, Washington D.C., 1979.

Snyder, C. W., M. Neugebaur, and U. R. Rao, The solar wind velocity and its correlation with cosmic ray variation and with solar and geomagnetic activity, J. Geophys. Res., 68, 6361-6370, 1963.

Sontakke, K. G., N. K. Thakur, and G. K. Rangarajan, Geomagnetic calm intervals and anomalous solar cycle 20, Proc. Indian Acad. Sci. (Earth Planet. Sci.), 91, 235-239, 1982.

Svalgaard, L., Geomagnetic activity: Dependence on solar wind parameters, in Coronal Holes and High Speed Solar Wind Streams, edited by J. B Zirker, pp. 371-441, 1977.

Vautard, R., P. Yiou, and M. Ghil, Singular spectrum analysis: a toolkit for short noisy chaotic signal, Physica, D58, 95-126, 1992.

Wang, Y. N. and N. R. Sheeley, Jr., The solar origin of long-term variation of the interplanetary magnetic field strength, J. Geophys. Res., 93, 1122711236, 1988.

Wang, Y.-M. and N. R. Sheeley, Jr., Solar wind speed and coronal flux tube expansion, Ap. J., 355, 726-732, 1990.

Webb, D. F. and R. A. Howard, The solar cycle variation of coronal mass ejections and the solar wind mass flux, J. Geophys. Res., 99, 4201-4220, 1994

Zeiger, B., Long term variations in pulsation activity and their relationship to solar wind velocity, geomagnetic activity and $F_{2}$ region electron density, J. Geophys. Res., 96, 21115-21123, 1991.

Zeiger, B. and K. Mursula, Annual variation in the near earth solar wind speed: Evidence for persistent north-south asymmetry related to solar magnetic polarity, Geophys. Res. Lett., 25, 841-844, 1998.

G. K. Rangarajan (e-mail: ranga@iig.iigm.res.in) and L. M. Barreto (e-mail: barreto@on.br) 